\title{
Is Science Really What Naturalism Says it is?
}

\author{
Federico Laudisa \\ Department of Human Sciences \\ University of Milan-Bicocca \\ federico.laudisa@unimib.it
}

\begin{abstract}
In spite of the relevance of a scientific representation of the world for naturalism, it is surprising that philosophy of science is less involved in the debate on naturalism than expected. Had the viewpoint of philosophy of science been duly considered, naturalism could not have overlooked the established lesson, according to which there is no well-defined recipe for what science must or must not be. In the present paper I address some implications of this lesson for (some forms of) naturalism, arguing that a radically naturalistic outlook fails to pay sufficient attention to some of the main lessons that philosophy of science has taught us concerning the nature of scientific theories. One of these lessons is that real scientific theories are far more normative than ordinary scientific naturalism is ready to accept, a circumstance that at a minimum is bound to force most naturalization strategies to re-define their significance.
\end{abstract}

Keywords: Naturalism; Normativity; Quine; Epistemology; Scientific Explanation.

DOI 10.1515/kjps-2017-0001

\section{Introduction}

A commonplace in the debates on naturalism is that there is no single core of assumptions that jointly characterize it uniquely. Daniel Andler, for instance, remarked that "philosophers have different views about the nature, structure and scope of naturalism, conceived as a very general stance towards human knowledge and the role played by the natural sciences" (Andler 2009, p. 284). Jaegwon Kim, in a work devoted to the 
American origins of naturalism put it more bluntly talking of a "plethora of naturalisms" (Kim 2003, p. 84), whereas in her book Second Philosophy Penelope Maddy turns ironical by noting that "the term 'naturalism' has acquired so many associations over the years that using it tends to invite indignant responses of the form 'but that can't be naturalism! Naturalism has to be like this!'” (Maddy 2007, p. 1). In general philosophical terms, we can take naturalism to be more an attitude than a rigorously defined claim, a sort of (at least) three-dimensional stance concerning respectively ontology, epistemology and the science/philosophy relationship. In its ontological dimension, naturalism accepts as possible true entities of the world only the sort of things that scientific theories posit as objects of their inquiry. In its epistemological dimension, naturalism holds that the methods employed by the scientific theories are the only methods that yield true knowledge. Finally, as far as the science/philosophy relationship is concerned, naturalism denies any privileged role for a philosophical conceptual analysis in the justification of knowledge itself. In turn, an epistemic form of naturalism may encompass further sub-variants, like a bio-inspired view of knowledge as a very fact of nature or those approaches that take scientific knowledge to be a special instance of the phenomena under investigation by cognitive science ${ }^{1}$.

Putting slight variations to the above framework aside, it can be hardly debated that naturalism has been the Zeitgeist in the analytic philosophy since the second half of the twentieth century. Dissenters are not absent ${ }^{2}$, but again Jaegwon Kim simply described an actual state of affairs when he wrote that "if current analytic philosophy can be said to have a philosophical ideology, it is, unquestionably, naturalism. Philosophical naturalism has guided and constrained analytic philosophy as its reign-

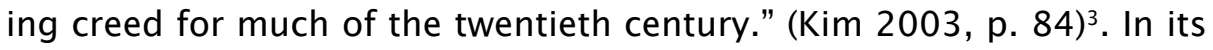

1 See for instance Giere 1999, Kovac 2007, Chandrasekharan, S., Nersessian, N.J. 2015.

2 Notable examples are such robust anti-naturalists as Alvin Plantinga or Michael Rae.

3 Even if we overlook the extent of the divergences between the worshippers of naturalism and its enemies, it is useful to point out a factor that turns out to be constitutive, although not always easy to characterize precisely in every domain: naturalism essentially relies on a model of knowledge that derives straightly from science and, more generally, on the role of paradigm of knowledge that science has been playing in the last three centuries. The central position of a certain image of scientific rationality in all variants of 
most influent and radical formulation, originally due to W.V.O. Quine, naturalism concerned mainly the theory of knowledge and found its expression in terms of what has been called replacement naturalism (Kornblith 1994a, Almeder 1998, Feldman 2012), according to which traditional epistemology is to be replaced by the scientific analysis of empirical processes underlying the formation of beliefs concerning the natural world ${ }^{4}$. On the very basis of the extent to which naturalism relies on a scientific representation of the world, however, it is surprising that the perspective of the philosophy of science is much less involved in the analysis of a naturalistic outlook than expected, outweighed as it is by the perspectives, say, of epistemology or philosophy of mind. Had the viewpoint of philosophy of science been taken more seriously into account when discussing the philosophical foundations of naturalism, a basic fact could not have been overlooked: namely, that in spite of the naturalism reliance on a scientifically-oriented paradigm of knowledge, one of the few established lessons of the philosophy of science of the twentieth century is that the question «What is science, exactly?» is far from settled in abstract and rigorous terms. There seems to be no set of necessary and sufficient conditions that determine the boundaries of a scientific theory, as opposed to a non-scientific one and this appears to have relevant implications for (scientific) naturalism, implications that the debate on naturalism does not seem always to take seriously. In her above mentioned book Second Philosophy, for instance, Maddy is among the few who recognize this circumstance as a potential difficulty that naturalism might have to face: in fact Maddy aims to pursue a new project that turns out to be still naturalistic - a project she qualifies as 'Second Philosophy' - but since "there is no hard and fast specification of what 'science' must be, [...] there can be no straightforward definition

naturalism implies then a special attention to a circumstance: that the scientific revolution has introduced into Western culture a sort of new category - that of having an existence according to science - that simply did not exist previously (Stein 1993) and that appears to decisively shape the whole subsequent philosophical investigation concerning ontology, epistemology and their complex relationships.

4 In the Quinean replacement naturalism, epistemology is reduced in principle to a very peculiar kind of 'science', namely behaviorist psychology. Whether the Quinean talk might be referring to all kind of science is a different question ( $\mathrm{am}$ grateful to a referee for this point). For a recent overview of the pre-Quinean history of naturalism in epistemology, the Quinean project and the wide array of reactions to it, see Rysiew 2016. 
of Second Philosophy along the lines 'trust only the methods of science'" (Maddy 2007, p. 1).

As a matter of fact, the claim according to which there is no set of necessary and sufficient conditions through which a scientific theory is to be uniquely characterized might be taken as the most general lesson that we learn from the overall development of philosophy of science in the XXth century, although the details of this claim are not as straightforward to formulate as one might hope and the intellectual processes leading to this outcome are complex. In this line of thought, we can safely refer to the philosophical work of T.S. Kuhn and W.V.O. Quine as the main sources for the claim that there is no recipe for what science must be: for both Kuhn and Quine one of the critical key points - if not the critical key point - is the empiricist assumption concerning the existence of a clear boundary between what counts as empirical and what counts as non-empirical within the body of a scientific theory. On the background of such an assumption, logical empiricists identified the task of the epistemology of science as that of providing a rational reconstruction of scientific theories in which the logical structure of the theory should have been a privileged outcome of the analysis: it is not surprising that in this enterprise of a rational reconstruction of scientific theories 'from outside', so to say, philosophy was supposed to play a major role.

By the viewpoint of such critics of this stance as Kuhn and Quine, the sort of work that philosophical analysis was supposed to perform was normative in itself, since the rational reconstruction of a scientific theory $T$ turned out to be a justification of how $T$ was ideally to be, rather than a de facto reconstruction of how $T$ was in practice. With special reference to Quine, his attack to the <theory/experience> distinction of logical empiricists was therefore largely consistent with the anti-normative bend of his version of naturalism, something that in turn is consistent with the naturalists' denial of any privileged role for a philosophical analysis in the justification of scientific theories. There seems to be, however, a philosophical tension here. What I would like to argue in the following is that if a scientific theory is far from being as easily read off its observational basis as logical empiricists hoped, this need not motivate a radically non-normative stance toward scientific theories. If no clear-cut boundary exists between empirical and non-empirical within a scientific 
theory, this may well pave the way for the idea that the construction of a scientific theory is also, to a certain extent, the outcome of a series of epistemic decisions, that in turn partly depend on a class of normative criteria (justifying, for instance, why should we prefer a theory with fewer primitive notions, with an elegant and non-contrived mathematical formalism, and so on). In this sense, blurring the dividing line between empirical and non-empirical in a Quinean spirit need not imply erasing any trace of normativity within scientific theories.

In the present paper, I will try to address some of these implications of the [no-recipe-for-what-science-must-be] result for naturalism as a general outlook. I will start in section 2 by focusing on the very significance of the distinction between ontological and epistemic naturalism. I will defend a sort of conceptual priority of the latter over the former, on the basis of which an epistemic form of naturalism might be taken in a way to 'embody' an ontological form of naturalism, a conclusion that strengthens the idea that in order to characterize what naturalism is one must be clear on what the nature of scientific theories is. This point leads to section 3, where I will focus on the role of scientific theories in what looks as a typical move of scientific naturalism, namely the so-called naturalization strategy. As a matter of fact, a large part of present philosophical frameworks inspired by scientific naturalism implicitly assume that when in the naturalizing strategies we move from a notion-to-be-naturalized toward science, there is a corresponding decrease of normativity. This process is taken exactly as one of the most desirable and sought-for aims of the strategies themselves: if the notion $X$ to be naturalized is a highly normative one - hence a notion that for this reason might appear at first sight hard to integrate into a scientific view of the world - the naturalization treatment is often taken to be a sort 'de-normativization' process. Part and parcel of my analysis will be to argue, on the contrary, that real scientific theories are far more normative than ordinary scientific naturalism is ready to accept, a circumstance that at a minimum is bound to force most naturalization strategies to re-define their significance. In section 4 I will address the Quinean proposal according to which the admittedly irreducible normativity within epistemology should be re-cast as a form of technology-of-truth-seeking: on the basis of some recent works in the philosophy of technology, I will argue that the outcome 
of this move in fact neither removes nor neutralize normativity within epistemology, since the notion of technology itself is bound to have a significantly normative dimension. In section 5 I will argue that a further proposal put forward by Larry Laudan in order to make normativity and a naturalistic philosophy of science compatible does not avoid the above mentioned tension: finally, in the last section I will draw some tentative, general conclusions.

\section{On the Mutual Independence of Ontological and Epistemic Natu- ralism}

In the debates on naturalism, there is a customary distinction between ontological naturalism and epistemic naturalism (see e.g. De Caro, Macarthur 2004, pp. 3-6). In the former case, ontological naturalism identifies nature as self-sufficient and identical to the totality of reality. Even before the well-known Sellarsian adaptation of Prothagoras' fragment - "in the dimension of describing and explaining the world, science is the measure of all things, of what is that it is, and of what is not that it is not" (Sellars 1963, p. 173) - already Ernst Nagel used to characterize interestingly ontological naturalism as follows:

In my conception of it, at any rate, naturalism embraces a generalized account of the cosmic scheme and of man's place in it, as well as a logic of inquiry [...]Two theses seem to me central to naturalism as I conceive it. The first is the existential and causal primacy of organized matter in the executive order of nature. This is the assumption that the occurrence of events, qualities and processes, and the characteristic behaviors of various individuals, are contingent on the organization of spatio-temporally located bodies, whose internal structures and external relations determine and limit the appearance and disappearance of everything that happens. That this is so, is one of the best-tested conclusions of experience. [...] The second major contention of naturalism is that the manifest plurality and variety of things, of their qualities and their functions, are an irreducible feature of the cosmos, not a deceptive appearance 
cloaking some more homogeneous" ultimate reality"or transempirical substance, and that the sequential orders in which events occur or the manifold relations of dependence in which things exist are contingent connections, not the embodiments of a fixed and unified pattern of logically necessary links. (Nagel 1956, pp. 8-9, italics in original; similar statements can be found for instance in Armstrong 1981, p. 149, Armstrong 1983, p. 82, and Kim 2003, p. 90).

On the background of modern science, formulations like these sound intuitive at first sight. There is a highly non-trivial problem underlying it, however. This problem is likely to affect any formulation of ontological naturalism since it has to do with the view, largely presupposed, according to which the task of ontological naturalism is to flesh out the metaphysical implications of scientific theories. This means that, according to this view, it is a scientific theory that obviously leads naturalism to qualify the existence of objects. But if it is a theory that provides a structure through which we are supposed to access reality, it follows that the ontological commitments are mediated by epistemic requirements of the theory itself, those requirements through which we are naturalistically entitled to say that there are really existing objects and structures. The objects of the theory - what the theory is about - are to a large extent constructs, let us call them $C$, characterized by a number of abstract conditions that the theory is supposed to assume in order to be truly a theory of the objects $C$. This feature largely goes along with the semantic view of theories typical of the post-positivistic philosophy of science (van Fraassen 1980, Suppe 1989), and with what has been called a contextual theory of the meaning for scientific terms: the way in which theoretical terms refer - even when they are assumed to genuinely refer - depends, often holistically, on the global structure of the theory itself (Holger 2013). Hence, if the ontological characterization of portions of natural reality essentially depends on epistemic constraints, what is known as ontological naturalism is likely to become more dependent on its epistemic counterpart than it is usually suggested: moreover, these epistemic constraints are not uniform since different theories structure their relevant portions of reality according to possibly different standards. 
Let us take into account in this direction, for instance, a passage from the above Nagel text, in which the author introduces the assumption that "the occurrence of events, qualities and processes, and the characteristic behaviors of various individuals, are contingent on the organization of spatio-temporally located bodies, whose internal structures and external relations determine and limit the appearance and disappearance of everything that happens". The spatio-temporal constraint is again, at first sight, entirely plausible if we speak of a natural reality, but it must also be stressed that in order to make sense it requires a precise and well-formulated theory of space-time, since - as Nagel claims - it is the set of structures and relations dictated by this theory that determine "the appearance and disappearance of everything that happens".

According to another eminent naturalist philosopher, Hilary Kornblith, the task of a naturalistic metaphysics is "simply to draw out the metaphysical implications of contemporary science [...] A metaphysics which goes beyond the commitments of science is simply unsupported by the best available evidence" (Kornblith 1994, p. 40). The wording of this formulation is especially apt to support the claim that the epistemic one is in fact the only non-derivative strand of naturalism. For if scientific theories fix the commitments that a naturalistic metaphysics - whatever it might be - cannot afford to transcend, this means that it is the wide class of epistemic structures that has a priority over the 'world' of ontological structures. This is further strengthened by the reference to the 'available evidence': under the hypothesis that metaphysics needs to be supported by scientific theories in order to be acceptable, the role that evidence might play in this support is highly theory-dependent, not to speak of the highly theory-dependent character of the very notion of evidence itself (a further lesson we have learned from the post-positivistic philosophy of science). In a vein similar to Kornblith, David Papineau claims that "the driving motivation for ontological naturalism is the need to explain how different kinds of things can make a causal difference to the spatiotemporal world" (Papineau 2009, emphasis added). Namely, Papineau stresses that what ontological naturalism is about (the 'content of reality', as Papineau puts it) must be formulated in terms of what makes a causal difference to the spatiotemporal world. The condition of 'making causal difference in spacetime' prescribes then the kind of 
property that an $x$ must exhibit in order for $x$ to be really an object of the natural world: but this is a causal requirement, which implies that (i) some theory of causation must be presupposed on the background, and (ii) it must take into account the circumstance according to which different theories may have wildly different causal requirements. Finally, in a recent paper devoted to the effort of defending a compatibility between a certain form of naturalism and the Husserlian phenomenology, Ramstead characterizes ontological naturalism as "the position that all things and their properties are natural things and properties, or supervene on natural things and properties" (Ramstead 2015). Once again, however, this metaphysically-tinted formulation hides a dependence from epistemic constraints, since it is a scientific theory - and not the Nature that tells us what is 'natural': as Ramstead himself claims two lines in advance, "natural stuff is the kind of stuff postulated by the ontologies of the natural sciences" and it is the relevant theory that decides what belongs to such an ontology and what does not ${ }^{5}$.

A notable example of the interplay between ontological and epistemic naturalism which is consistent with the picture I have outlined above is the interpretational debate on the foundations of quantum mechanics. This debate is a lively illustration of the circumstance in which, in order to characterize the nature of the reality that is the intended target of the theory, we have to address a wide disagreement on how a theory suitable for that purpose should be formulated. If, on the one hand, quantum mechanics qualifies as a cornerstone in the description of the ultimate nature of physical reality and hence in the perspective of ontological naturalism, on the other hand there is no consensus on the very issue of what is it exactly that the theory is about. According to some, there was not even a problem with 'interpreting' the theory: the great nuclear physicist Rudolf Peierls once said that "the use of the term Copenhagen interpretation sounds as if there were several interpretations of quantum mechanics. There is only one. There is only one way in which you can understand quantum mechanics [...] so when you refer the Copen-

$5 \quad$ To Werner Heisenberg, who was arguing that "a good theory must be based on directly observable magnitudes", Einstein aptly replied: "It is the theory which decides what we can observe" (Heisenberg 1971, p. 63). 
hagen interpretation of the mechanics what you really mean is quantum mechanics». (Peierls 1986, p. 71). In a more recent paper in a similar spirit, a paper with the eloquent title Quantum theory needs no 'interpretation', Fuchs and Peres have claimed that "quantum theory does not describe physical reality. What it does is provide an algorithm for computing probabilities for the macroscopic events ('detector clicks') that are the consequences of our experimental interventions. This strict definition of the scope of quantum theory is the only interpretation ever needed, whether by experimenters or theorists.» (Fuchs, Peres 2000, pp. 71-72). On the contrary, in the so-called observer-free formulations of (non-relativistic) quantum mechanics like Bohmian mechanics or dynamical-collapse models, quantum mechanics describes either quantum particles or matter fields in physical space and time, in a framework in which this space-time ontology is the primitive ontology and the measurement-related processes are derivative ${ }^{6}$.

In more general terms, a dilemma seems then to arise for a generally naturalistic stance: either we assume metaphysical hypotheses unrelated to science, with the consequence that we are likely to transcend the ordinary boundaries of naturalism, or we accept that the metaphysical hypotheses are in effect constrained by what scientific theories tell us concerning the items involved in those hypotheses, with the consequence that a genuinely 'ontological' naturalism is bound to be hardly distinguishable from an epistemic one. Two points must be stressed, however. First, the claim according to which any ontological naturalism is bound to strongly depend on epistemic naturalism has no anti-naturalistic tone per se. Second, the alleged dependence of ontological naturalism on epistemic naturalism need not imply reduction or elimination of the former. We might want to preserve a place for ontological naturalism in the logical space by assuming that - in deciding what is an object for natural sciences - there can be a sort of 'pre-comprehension' of natural reality, something that tends to include certain items and to exclude others: this pre-comprehension would concern items like mat-

$6 \quad$ There is now an extended literature on the details of such an ontology and its connections with many of the general, interpretive issues in the foundations of non-relativistic quantum mechanics, and this is not the place to provide a review. For a recent presentation and re-assessment, see Dürr, Goldstein, and Zanghì (2013). 
ter, space, time, causation and the like, and would be conceived of as an intrinsically metaphysical character, independent of the specific scientific formulations of these notions. According to Horgan and Timmons, for instance "we take the naturalist outlook in philosophy to be at bottom a metaphysical view about the nature of what exists. The vague, pre-theoretic idea the philosophical naturalist attempts to articulate and defend is that everything - including any particulars, events, facts, properties, etc. - is part of the natural physical world that science investigates" (Horgan, Timmons 1993, p. 182, emphasis added), although it might still turn out more difficult that it seems to accommodate a metaphysical hypothesis in a naturalistic framework, if not through the mediation of an epistemic structure like a scientific theory (MacLaurin, Dyke 2012).

\section{The Role of Scientific Theories in the Naturalization Strategy}

Whatever the plurality of naturalisms, there is a typical move in the scientific brand of naturalism: the so-called naturalization strategy. Just like what happens for the very definition of naturalism itself, we can interpret also a naturalization strategy in several different ways, according to the different tasks such a strategy is supposed to perform, and also according to whether we think that such a strategy is advisable or not! Once again, however, a common feature can be discerned, all this variety notwithstanding: a feature according to which a naturalization strategy works as a sort of decrease-of-complexity tool, namely a tool adopted with respect to a certain philosophical notion or issue $A$, when $A$ is assumed to be 'intractable' to a serious extent, that is too dependent on subjective, contextual, normative factors and the like.

Although expressed in admittedly vague terms, this formulation of (the core of) a possible naturalization strategy is in line with ordinary descriptions of what is the effort in which a naturalistic attitude is supposed to engage in this or that area of investigation. In a review paper on the project of naturalizing semantics, for instance, Barry Loewer describes in these terms the crucial issue under discussion:

The semantic properties of mental states are what makes them intentional states. Thus the intentional content of e.g. the thought that the cat is on the mat is the truth conditions 
of the thought. The topic of this paper is the question: In virtue of what do intentional mental states/events possess their semantic properties? For example, what makes it the case that a particular thought is about the cat and has the truth conditions that the cat is crying? The answer cannot be the same as for natural language expressions since the conventions that ground the latter's semantic properties are explained in terms of the semantic properties of mental states. If there is an answer, that is, if semantic properties are real (and really instantiated) and are not fundamental, then it appears that they must be instantiated in virtue of the instantiation of certain non-semantic properties. (Loewer 1997, p. 108).

Still in Loewer's words, the project of the philosophers he calls "Semantic Naturalizers" is to address the above issue in a naturalistic vein: "Semantic Naturalism's central contentions are a) that semantic properties, laws, causal relations involving them obtain in virtue of the obtaining of facts constituted entirely by naturalistic properties etc. and b) that semantic properties are kinds of the sort suitable for investigation by the methods of the natural sciences."

By attempting to isolate the 'naturalistic properties' that should ground semantic properties, the naturalization strategy for semantics displays an element that I take to be common to most variants of naturalism: its being essentially a simplifying strategy. That is, what explicitly or implicitly a naturalization strategy usually tries or hopes to do is to select a specific scientific domain, that is supposed to play the role of the 'simplified' domain in terms of which the notion A might be fruitfully reformulated: after this naturalization treatment, clearly, we expect the complexity of $A$ to have been drastically reduced.

But what do we mean by 'complexity' here? Although at this stage I am not in the position of characterizing it in very rigorous terms, I claim that a plausible way to interpret this notion of complexity is essentially in explanatory terms. For, in principle, a naturalization strategy might also imply in certain respects an increase of complexity: a neurobiological theory of a mental function might be taken to reduce the types of sub- 
stances that we assume to belong to the natural realm, but on a strictly theoretical level it might well be significantly more complex than the folk counterpart notion that is supposed to replace, not to speak of the hypothesis in which one should attempt to give - say - a microphysical description of the neurobiological structures themselves. But if we focus on explanatory complexity, then the idea according to which the naturalization strategy is a complexity-reducing strategy makes much more sense: in a naturalistic framework, a neurobiological theory of a mental function - although theoretically more sophisticated - is supposed to assimilate the explanation of that function to other, more typically neurobiological kinds of explanation, under the assumption that the world to which these explanations refer to is decisively simpler to account for than the world to which folk-psychological explanations refer to.

For the sake of the argument let us assume for the moment that this simplification attempt suitably represents one of the most pressing ambitions of naturalism. If by $A_{S D \text {-Nat }}$ we denote the notion $A$ after its naturalization in terms of the scientific domain $S D$, this attempts should reasonably satisfy something like the two following requirements: first, the explanatory complexity of $A_{S D-N a t}$ should be significantly less than the explanatory complexity of the original, non-naturalized $A$; second, the explanatory complexity of $A_{S D-N a t}$ must be 'comparable' to the explanatory complexity of the notions that typically $S D$ itself is able to control. Under the hypothesis that we are able to master the explanatory complexity of the central notions of $S D$, if the $S D$-naturalization of $A$ failed to satisfy these two requirements, then it would not result in a significant gain in making the notion $A$ 'more tractable'. Should the first not hold, there would be no point in naturalizing $A$ in the first place, whereas should the second fail to hold, we could reduce the explanatory complexity of $A$ but only to an 'insufficient' extent: the naturalization would be unsatisfactory anyway, since our original point was to 'translate' $A$ in terms of notions that the scientific domain $S D$ does master. As far as philosophy of science is concerned, it is this explanatory complexity-reducing role that - in my view - has been an actively operating factor for the attractiveness of naturalism. With a geometrical analogy, we might liken this naturalistic reduction of complexity to a projection operation by which we map, say, a four-dimensional object onto a three-dimensional space, so as to make 
it available to visualization: the very fact that after the projection we can visualize an object that we were unable to conceive previously would testify that some sort of cognitive simplification has been obtained. Moreover, the complexity-reducing role leads one to hope that it might be science that is called to do the 'dirty job' about issues, for instance, like the mind-matter relation, intentionality, meaning and many others, deciding once and for all whether there might be an ultimate answer in scientific terms to thorny issues like $<$ Is there anything to the mind but matter?>, <Can a computer really catch meanings?>, <Can an artificial cognitive system extract semantics from syntax? $>$, etc.

This emphasis suitably resonates with how naturalization is usually conceived. In his above mentioned work, Ramstead characterizes naturalization as a true paradigm shift:

[...] to naturalize a thing entails that one mobilizes only those concepts that pertain to the ontologies of the natural sciences to explain a given phenomenon, and to abandon those concepts that were previously used to account for it which are not part of the lexicon of the natural sciences [...] I propose, then, to read the expression 'to naturalize a thing or property' throughout as meaning 'to give an explanatory account of a thing that is coherent with the ontologies of the natural sciences'. It is thus a manner of speaking about a change in our conceptual or semantic network with regard to a thing or property that was heretofore not conceptualized as a natural one. (Ramstead 2015).

Should the attempt of giving 'an explanatory account of a thing that is coherent with the ontologies of the natural sciences' fail to simplify the complexity of such possible account, or even keep it essentially the same (not to speak of increasing it), I strongly doubt that the naturalization strategies would have deserved the appeal they in fact had (see also Wright 2007, pp. 585 ff).

If for the sake of argument we assume the above characterization of the naturalization strategies, we must note a further element that the complexity-reducing procedures of the different naturalization strat- 
egies attempt to reduce: the amount of normativity inherent to the not-yet-naturalized notion $A^{7}$. In principle, this attempt looks perfectly reasonable in the overall strategy, since the difficulty of suitably integrating normative notions into a scientific view of the world is in itself an addition of complexity that a naturalistic outlook might wish to reduce, eliminate or confine. Although reasonable, however, this 'de-normativization' carries with itself a problem, whose potential is once again more apparent if we agree to consider it from the viewpoint of the philosophy of science. For the naturalization strategy, with all its promises of simplifying matters, is far too optimistic over the possibility that when we proceed from the not-yet-naturalized $A$ to the $S D$-naturalized $A$ (what we indicated above by $A_{S D \text {-Nat }}$, for some scientific domain $S D$ ), we actually follow a path that starts from a highly normative domain and ends to a significantly less normative domain, or hopefully non-normative at all: in other words, the scientific domain $S D$ might be much more controversial than expected as to what it takes to explain satisfactorily the naturalized notion $A_{S D \text {-Nat }}$. The problem is simply that the expression 'having a scientific explanation' is strongly theory-dependent and far from having a unique meaning. Different theories have different modalities of taking their pertaining phenomena to be 'explained' within their frameworks: sometimes, a theory may claim to have 'explained' a class of phenomena just because there it possesses a predictively effective model or because a correlation with more familiar phenomena has been established, whereas other theories will require much more. In his Beyond Reduction (2007) Steven Horst voices a similar worry with regard to naturalization strategies in philosophy of mind. After defining a general 'naturalistic schema' about a domain $D$ as "the view that all features of $D$ must be accommodated within the framework of nature as it is understood by the natural sciences" (Horst 2007, p. 13), he aptly remarks that "even once we have pinned down what we mean by 'accommodating' the mind within nature, the expression 'the framework of nature as it is understood by the natural sciences' is still rather vague. Just what our

$7 \quad$ The role of normativity within a scientifically-inspired naturalism is of course a hotly debated issue and the relative amount of references is remarkable. Here we limit ourselves to refer the reader to two collective works on the subject: Braddon-Mitchell, Nola (2009) and De Caro, Macarthur (2010). 
naturalistic schema means will depend heavily upon what one considers to be central to how the natural sciences operate, and how they represent the natural world." (Horst 2007, pp. 14-15, emphasis added) ${ }^{8}$.

Therefore, even provided the de-normativization procedure allegedly inherent in the naturalization strategy is held to be possible, this might be not without cost, since such procedure might obscure the role that normativity appears to play anyway within scientific theories 9 . Moreover, in addition to recognizing that the scientific domain that is supposed to host the post-treatment notion $A_{S D \text {-Nat }}$ is significantly more normative than hoped by many die-hard naturalists, there is also the problem of how the naturalization strategy is supposed to work. In fact, any naturalization strategy is not pursued in a vacuum but according to a more or less well-defined set of criteria, that shape the particular ways in which the notion $A$ gets translated into the notion $A_{S D-N a t}$ : now, these criteria turn out to be to a large extent normative and hardly naturalizable themselves.

\section{The Quinean ‘Normativity’ of Epistemology}

The ambivalence on the role of normativity w.r.t. the construction of scientific theories can be found not only in interpreters but within the

8 With respect to a physicalistic variant of naturalization, for instance, it seems similarly hard to provide a convincing reply to the following question: "How can we ask our fundamental physical theories to tell us about what there is in the world, when each of those theories is subject to multiple interpretations, interpretations that often radically disagree with one another about what kind of a world the fundamental theory is really describing?" (Sklar 2001, p. 47). For a discussion on normative criteria concerning the adoption of a given interpretation in the area of the foundations of quantum mechanics, see Guillermin, Dedeurwaerdere (2013).

9 By exploiting again a geometrical analogy, it seems that we deal here with a sort of epistemic counterpart of a theorem proved by Georg Cantor, who proved that there is an isomorphism between the set of points of a square $Q$ (excluding the points lying on the sides) and the set of points of anyone of the sides of $Q$ : put simply, a square has provably as many points as any of its sides. This means that we can establish a one-to-one correspondence between a two-dimensional object ( $Q$ minus its sides) and a one-dimensional object (one of the sides of $Q$ ) - so in a sense we reduce the complexity of $Q$ - but, when we consider the passage from two dimension to one, we pay the price of destroying the form of the square, that is what constitutes in a sense the very geometrical identity of the object 'square'. 
very Quinean philosophical system. On the one hand, Quine is one of the chief responsible for the failure of the logical empiricist hope of a recipe for what science must be, since the Quinean attack in Two Dogmas turned out to be fatal to the above mentioned hope. A key role in this attack is played by the notion of under-determination, which curiously enough shows up simultaneously with the Quinean tendency to underestimate the role of normativity within scientific theories. In fact, in a well-known passage of "Epistemology Naturalized", namely the very birthplace of the twentieth-century naturalism vis-à-vis the philosophy of science, Quine writes:

Epistemology, or something like it, simply falls into place as a chapter of psychology and hence of natural science. It studies a natural phenomenon, viz., a physical human subject. This human subject is accorded a certain experimentally controlled input - certain patterns of irradiation in assorted frequencies, for instance - and in the fullness of time the subject delivers as output a description of the three-dimensional external world and its history. The relation between the meager input and the torrential output is a relation that we are prompted to study for somewhat the same reasons that always prompted epistemology: namely, in order to see how evidence relates to theory, and in what ways one's theory of nature transcends any available evidence (Quine 1969, pp. 82-3).

In the Quinean reflection on the nature of scientific theories, the meager input-torrential output relation is exactly the relation that malgré soi is apparent in under-determination: in its being 'torrential' the output far outweighs what the 'meager' input strictly allows. It is exactly under-determination that makes room for normativity even within the Quinean framework, which is the naturalistic project par excellence: if our scientific theory "transcends any available evidence", then we will have to adopt some set of possible criteria in order to strengthen it, and at least some of these criteria will be inevitably normative in nature. This consequence is inherent in a detachment from the logical empiricists' project and their views on the role of observation in science: as Quine wrote in a further 
passage "the most modest of generalizations about observable traits will cover more cases that its utterer can have had occasion actually to observe. The hopelessness of grounding natural science upon immediate experience in a firmly logical way was acknowledged" (Quine 1969, p. 74).

On the other hand, in the passage above on the meager input-torrential output relation, Quine seems also to assume without further argument that scientific evidence is completely fixed - in a sort of functional way by the several causal relations between perceptual input and theoretical output. There seems to be a tension here. Exactly due to the pervasive phenomenon of the under-determination so vividly represented by Quine himself, the reason why a class of perceptual inputs works as evidence for a certain theory clearly exceeds the mere causal relations that the perceptual inputs may establish with bits of the theory, and depends also on the normative decisions taken by the subjects involved in the evaluation whether that class can be really treated as evidence or not. Moreover, it is the very notion of evidence that cannot be entirely deprived of a normative dimension, even in the Quinean framework. In other terms, that a certain class of perceptual inputs does or does not constitute evidence for the plausibility of a given theory or model cannot simply read off the causal relation between those inputs and a given output: for an event $e$ to provide 'evidence' for a theory $T$ is something that decisively depends on epistemic choices on the part of the subjects involved, something that in turn is the effect of a largely normative activity. To the extent to which the notion of evidence is connected to the general idea of justification, the de-normativization of evidence leads in fact to the elimination of justification itself from epistemology, an elimination that according to many is likely to deprive knowledge itself of any meaning. Curiously enough, this argument can be further supported with the aid of a sort of 'naturalistic' remark: since the different biological species employ different nomological patterns to connect inputs and output but the input-output relation must remain evidential in order to be scientifically significant, the notion of evidence is to retain a normative character that abstracts from the details of the above mentioned patterns ${ }^{10}$.

10 See e.g. Kim 1988, pp. 389-90. In their influential paper "Saving the Phenomena", Bogen and Woodward put forward a distinction between data and phenomena and claim 
To be true, Quine did not ignore altogether the issue of normativity within the framework of his naturalistic epistemology, but proposed a sort of 'weak' version of it according to which normativity is accounted for in terms of predictive efficacy.

Naturalization of epistemology does not jettison the normative and settle for the indiscriminate description of ongoing procedures. For me normative epistemology is a branch of engineering. It is the technology of truth-seeking, or, in a more cautiously epistemological term, prediction. [...] The normative here, as elsewhere in engineering, becomes descriptive when the terminal parameter is expressed. (Quine 1986, pp. 664-5).

In Quinean terms, the normative side of epistemology lies in the selection of the tools that should turn out to be the most effective in improving the predictive performances of science, with the aim of making optimal the above mentioned meager input-torrential output relation. Although weak, however, this formulation does not stand less in need of explanation: even if we admit that 'normativity' here reduces to a sort of checking procedure for the plausibility of certain predictive strategies, who or what legitimates that procedure? How and to what extent can the normative really turn descriptive?

But even if we leave aside for the moment these rather general questions and we assume to take somewhat seriously the terms engineering and technology, the question remains of how exactly these terms are to be interpreted and of whether this interpretation really allows one to have an innocuously 'normative', but truly naturalistic epistemology. A problem that immediately arises is whether the adoption of the technology-of-truth-seeking viewpoint can really explain away normativity in

that what the theories are supposed to explain are facts about phenomena - which are ideal constructs - and not about data which, after all, are the only observable part of science (Bogen, Woodward 1988). The data provide evidence for the phenomena but, according to the Bogen-Woodward distinction, the way in which phenomena are "constructed out from data' is admittedly complex. In this perspective, we readily acknowledge that in the rational space that is opened between data and phenomena we happen to employ normative criteria. 
epistemology, something that appears to be the main aim of the Quinean proposal. In this respect, a simple look at the common characterization of technology shows a conceptual tension, with relevant philosophical implications: according to a widespread view, "technology is a practice focused on the creation of artifacts and, of increasing importance, artifact-based services" (Franssen, Lockhorst, van de Poel 2015). So, given the centrality of the notion of artifact within this view, we may well wonder what are the artifacts this sort of engineering-type of epistemology deals with. According to a plausible option in this particular context, the kind of artifact epistemology might be taken to be concerned with is what Houkes called an epistemic engine, "i.e. a device that is able to navigate its surroundings and to anticipate situations. The «epistemological engineer» can specify rules for the proper use of this artifact, which might be understood as either the human subject or its brain." (Houkes 2003, p. 261).

Even if couched in a philosophical terminology, epistemic engines share with all other artifacts what has been called their dual nature: on one hand artifacts are physical systems, on the other hand they are structures conceived to realize functions whose significance is fixed by a complex, intentional framework, namely a framework of meanings, wishes, choices, plans and all that contributes to provide portions of the natural world with a functional meaning within well-defined socio-cognitive contexts (Kroes, Meijers 2006). It is with reference to this framework, for instance, that the notions of design plays its essential role for technology: "The design process, the structured process leading toward that goal, forms the core of the practice of technology" (Franssen, Lockhorst, van de Poel 2015). The dual nature of artifacts, then, is in general terms simply another dimension of the problem of combining the natural world with the intentional world, but more in particular is also something that makes turning epistemology as engineering of knowledge into a purely descriptive enterprise an extremely hard task to accomplish. Not only because the kind of intentionality that must be assumed according to the dual nature of artifacts resists naturalization, but also because of what Hand Radder has called the 'inherent normativity of technology'. Radder characterized technologies as "artifactual, functional systems with a certain degree of stability and reproducibility" (Radder 2009, 
p. 897): namely, technologies give rise to aim-related artifacts which are supposed to last in efficiency for a reasonably long period of time. According to Radder, an important implication of this characterization is that "technologies are inherently normative, because their stable and reproducible realization in some region of space and time requires that the people in that region should behave in such a way as to enable, and not disturb, the intended functioning of the technology." (Radder 2009, p. 897). This means that, in this framework, normativity is an essential ingredient of technology both at its initial and final stage: at the former, since technological artifacts are conceived of in an intentional framework in which normative factors play a decisive role; at the latter, since a successful realization of technologies requires from the (psychological, social, cultural) intended environment for them the acceptance of a system of norms, in order for these technologies to fully display their potential. If we go back to the Quinean 'engineering of knowledge' view and suppose to translate the above argument in epistemological terms, the 'artifact' - according to the Houkes proposal - is an epistemic engine and the environment in which such artifacts are supposed to display their activity is essentially nothing but the scientific community. It seems then that the above argument can be replicated: at the beginning of the process, the framework in which an epistemic engine is conceived of as an artifact is an intentional one, in which aims, meanings and similar factors operate as normative constraints; at the other end, similarly, as long as epistemology is treated as a truth-seeking technology, the members of the scientific community to which epistemic engines are addressed are supposed to share epistemic norms in order for the epistemic-engines-as-truth-seekers to fully display their knowledge potential.

\section{Laudan Normativity: a Way Out?}

A further attempt to claim that normativity can happily coexist with a naturalistic epistemology has been proposed by Larry Laudan. He focused on the nature of those methodological rules ("Prefer theories allowing new predictions", "Prefer theories that can unify several existing theories", and so on) that are implicit in the development and the concrete construction processes of scientific knowledge. Within a framework that 
has been defined as meta-epistemological (or meta-methodological), in which "a denormativization of methodology is not entailed by its naturalization" (Laudan 1987, p. 25), Laudan's proposal is to reformulate such rules as hypothetical imperatives that establish relations linking epistemic means and ends:

Specifically, I believe that methodological rules, once freed from the elliptical form in which they are often formulated, take the form of hypothetical imperatives, whose antecedent is a stamen about aims or goals, and whose consequent is the elliptical expression of the mandated action. Put schematically, methodological rules of the form:

(0) "One ought to do $x$ "

should be understood as having the form:

(1) "If one's goal is $y$, then one ought to do $x$ ". (Laudan 1987, p. 24).

The main advantage of this reformulation, according to Laudan, lies in transforming methodological rules from pure prescriptions ('one ought to do $x^{\prime}$ ) in proper statements concerning the plausibility of certain means in view of certain cognitive ends ("If one's goal is $y$, then one ought to do $x$ ".). In principle, this move would achieve two points: (i) it makes explicit that a methodological rule is always contextually linked to a specific cognitive goal; (ii) it replaces expressions without a truth value into statements that, although preserving a prescriptive character, turn out to depend on how the world is made. If I adopt a rule like (1), that is, I assume implicitly that doing $x$ will contribute effectively to achieving $y$; but if this is true, then methodological rules turn out to be empirical statements, subject in principle to testing exactly like any other empirical statements: "methodological rules, on this view, are a part of empirical knowledge, not something wholly different from it" (Laudan 1987, p. 24). What Laudan proposes, then, is "the sketch of a naturalistic theory of methodology which preserves an important critical and prescriptive role for the philosopher of science, and which promises to enable us to choose between rival methodologies and epistemologies 
of science" (Laudan 1987, p. 29, emphasis in the original). Laudan's proposal aims to be normative, since the analysis of its object - the set of the methodological rules involved in the construction of scientific knowledge - requires an interpretive activity; but is meant to be also naturalistic, since the above rules owe their identity and their role to the certain states of affairs in the world: in this sense, rules themselves evolve not unlike the way in which scientific theories do (Laudan 1990, p. 46; cfr. also Kitcher 1992, pp. 69 ss.).

Several are the problems that Laudan's proposal has to face. First, Laudan admits that, in the evaluation of which are the best means to achieve a given cognitive end, there is a wide room for theoretical choices that can hardly be naturalized, but fails to explain how this can coexist with the naturalistic attitude of the whole enterprise. It is not by chance that the Laudan paper ends by arguing for the necessity to develop an axiology of research: "we need to supplement methodology with an investigation into the legitimate or permissible ends of inquiry" (p. 29). It is far from clear, however, what is the naturalistic grounding of such an axiology (Doppelt 1990, pp. 4-5, Knowles 2002, pp. 173-4). Second, even if for the sake of argument we assume that methodological rules are reformulated as hypothetical imperatives, namely as statements concerning relations between epistemic means and ends, the decision on what must be an epistemic end and why cannot entirely depend on how the world is made empirically, but once again on theoretical, hence conventional factors that resist complete naturalization ${ }^{11}$. Finally, in claiming that "the naturalistic epistemologist [...] construes epistemic claims as theories or hypotheses about inquiry, subject to precisely the same strategies of adjudication that we bring to bear on the assessment of theories within science or common sense" (Laudan 1990, p. 45), Laudan seems to be ready to defend the strong claim according to which also epistemic facts - as the modalities in which methodological rules govern the relations between epistemic means and ends - are really natural facts: it is a position that has been called substantive naturalism (Feldman 2012). In the perspective we are concerned with here, such a position might be invoked in order to support the claim that, when a class of possible

11 Siegel 1990; for a defense of Laudan on this point see Freedman 1999. 
criteria for constructing a theory (or for constructing a theory+interpretation) are involved, it should be possible (and desirable) to treat the very use of these criteria as a natural fact itself. As stressed by Hilary Kornblith, a naturalistic epistemologist who defends this kind of claims, "the subject matter of epistemology is knowledge itself, not our concept of knowledge" (Kornblith 2002, p. 1). In an earlier paper Hilary Kornblith had argued that "we must now try to explain how creatures with the faculties cognitive science tells us we have could have come to understand the kind of world which the sciences generally tell us that we inhabit" (Kornblith 1994, p. 43), on the background of the philosophical claim according to which human knowledge is a "natural phenomenon"12. Typical epistemic notions like beliefs are treated as the output of Darwinian evolutionary processes: "The various information-processing capacities and information-gathering abilities that animals possess are attuned to the animals' environment by natural selection, and it is thus that the category of beliefs that manifest such attunement - the cases of knowledge - are rightly seen as a natural category, a natural kind." (Kornblith 2002, pp. 62-3). In this framework, then, a major task is to find what Kornblith calls a theoretical unity underlying all those natural phenomena that should be characterized as 'cases of knowledge'. The formulation of such a theoretical unity, however, will require the use of certain criteria of classification, concerning what falls under the label case-of-knowledge and what does not. Are these criteria 'natural' (or 'naturalizable')? A further dilemma seems to arise at this point. If the criteria are natural, it seems that we can hardly interpret knowledge-as-natural kind still as knowledge in a philosophically meaningful way (Bonjour 2006). Moreover, there seems to be a threat of infinite regress. For it seems plausible that there might be different possible sets of criteria according to which one should collect all the 'cases of knowledge' under a 'theoretical unity: second-order criteria are needed, then, but they might not be unique as well, and so on. If on the other hand we suppose that the criteria are not natural, then it is far from clear how and to what extent we are still dealing with a naturalistic epistemology.

12 "I see the investigation of knowledge, and philosophical investigation generally, on the model of investigations of natural kind» (Kornblith 2002, p. 10-11). 


\section{Conclusions}

In the preceding pages I have attempted to point out a relevant gap in the debate on the scope and motivations of a naturalistic outlook, namely the lack of a sufficient attention to some of the main lessons that philosophy of science has taught us concerning the nature of scientific theories: given the role that naturalism attributes to science in framing the scientific image of the natural world, this lack appears to be hard to accept. Even more so if we consider that naturalization strategies can hardly underestimate how far from trivial a satisfactory characterization of a scientific theory can turn out to be (including the amount of epistemic normativity inherent in the theory itself). This conclusion need not justify a straightforward anti-naturalistic stance: it would be rather awkward to draw such an implication from a plea to pay a more serious attention to what philosophy of science is telling us. More simply, the caution we must have in remembering that when we talk about 'science' we actually talk about scientific theories is an additional argument against the rather extreme claim according to which philosophy should essentially 'dissolve into' science. By a strictly Quinean viewpoint, this dissolution is what should happen, because it would counteract once and for all the alleged tendency of philosophy to occupy a vantage point, from which to look at science 'from above' - so to say ${ }^{13}$. As a matter of fact, this 'dissolving' strategy gives rise to a further dilemma between a radical form of naturalism and a soft one, a dilemma that seems at the heart of the very significance of the whole naturalistic enterprise.

If we endorse a radical form of naturalism, by adopting the above dissolving strategy, philosophy seems to lose its legitimacy in trying to contribute to the analysis of the foundations of scientific theories - exactly the sort of analysis that looks like the very raison d'être of philosophy with respect to science. If on the other hand we endorse a soft form of naturalism, in which philosophy does not dissolve into science, we have

13 I must confess that I have never able to make sense of this sort of topological ('from-above') kind of accusation: the acknowledgement that within science there might be notions or issues that resist naturalization, hence open to an irreducibly philosophical analysis, need not imply that this analysis is supposed to be 'privileged' or 'more fundamental' in any sense. 
no crisp criteria in order to define a rigorous boundary that helps us to see where science ends and philosophy begins. Naturalism seems then to reduce here to the common sense recipe according to which philosophers, when they talk about notions or issues that might intersect science in one way or another, have to take into due account what specific scientific theories have to say concerning those notions and issues. A very reasonable advice indeed, but nothing that characterizes uniquely a naturalistic approach.

\section{References}

Almeder R. 1998, Harmless Naturalism. The Limits of Science and the Nature of Philosophy, Open Court, Chicago \& La Salle, Ilinois.

Andler D. 2009, "Is naturalism the unsurpassable philosophy for the sciences of man in the 21 st century?", in Stadler, F., Hartmann, S., Dieks, D., Gonzalez, W.J., Uebel, T., Weber, M. (eds.), The Present Situation in the Philosophy of Science, Springer, Berlin, pp. 283-304.

Armstrong D. 1981, The Nature of the Mind and Other Essays, Cornell University Press, Ithaca N.Y.

Armstrong D. 1983, What is a Law of Nature?, Cambridge University Press, Cambridge.

Bird A. 2005, "Naturalizing Kuhn", in Proceedings of the Aristotelian Society 105, pp. 109-27.

Bogen J. Woodward J. 1988, "Saving Phenomena", The Philosophical Review 97, pp. 303-352.

Bonjour L. 2006, "Kornblith on knowledge and epistemology", in Philosophical Studies 127, pp. 317-335.

Braddon-Mitchell D., Nola R. (eds.) 2009, Conceptual Analysis and Philosophical Naturalism, MIT Press, Cambridge MA.

Chandrasekharan, S. \& Nersessian, N.J. 2015, "Building cognition: the construction of computational representations for scientific discovery" Cognitive Science 39, pp.1727-1763.

De Caro M., Macarthur D. (eds.), 2004, Naturalism in Question, Harvard University Press, Cambridge MA. 
De Caro M., Macarthur D. (eds.), 2010, Naturalism and Normativity, Columbia University Press, New York.

Doppelt G. 1990, "The Naturalist Conception of Methodological Standards in Science: a Critique”, in Philosophy of Science 57, pp. 1-19.

Dürr D., Goldstein S., Zanghì N. 2013, Quantum Physics without Quantum Philosophy, Berlin, Springer.

Feldman R. 2012, "Naturalized Epistemology", in E.N. Zalta (ed.), The Stanford Encyclopedia of Philosophy, <http://plato.stanford.edu/ archives/sum2012/entries/epistemology-naturalized/>.

Franssen M., Lokhorst G.J., van de Poel I. 2015, "Philosophy of Technology", in E.N. Zalta (ed.), The Stanford Encyclopedia of Philosophy, <http://plato.stanford.edu/archives/fall2015/entries/technology/>.

Freedman K. 1999, "Laudan's naturalistic axiology", in Philosophy of Science 66 (Proceedings), pp. S526-S537.

Fuchs C.A. , Peres A.2000, "Quantum theory needs no 'interpretation' ", Physics Today, March issue, pp. 71-72.

Giere R., 1999: Science without Laws, University of Chicago Press, Chicago.

Guillermin M., Dedeurwaerdere T. 2013, “Disagreement, Bell's Inequalities and Realism: The Role of Epistemic Values in Contemporary Approaches to Quantum Mechanics", paper available at Social Science Research Network: http://ssrn.com/abstract $=2284104$.

Heisenberg W. 1971, Physics and Beyond, Harper, New York.

Holger A. 2013, "Theoretical Terms in Science", in E.N. Zalta (ed.), The Stanford Encyclopedia of Philosophy, <http://plato.stanford.edu/ archives/sum2013/entries/theoretical-terms-science/>.

Horgan T, Timmons M. 1993, "Metaphysical Naturalism, Semantic Normativity, and Meta-Semantic Irrealism", Philosophical Issues Vol. 4, Naturalism and Normativity, pp. 180-204.

Horst S. 2007, Beyond Reduction, Oxford University Press, Oxford.

Houkes W., 2002: "Normativity in Quine's naturalism: the technology of truth-seeking?", in Journal for General Philosophy of Science 33, pp. 251-267. 
Kim J., 1988, "What is 'naturalized epistemology'?", Philosophical Perspectives 2, pp. 381-405.

Kim J. 2003, "The American origins of philosophical naturalism", in Journal of Philosophical Research, APA Centennial Volume, pp. 83-98.

Kitcher P. 1992, "The naturalists return", in The Philosophical Review 101, pp. 53-114.

Knowles J. 2002, “What's Really Wrong with Laudan's Normative Naturalism", International Studies in the Philosophy of Science 16, pp. 171-186.

Kornblith H. 1994a, "Introduction: What is Naturalistic Epistemology?", in Kornblith H. (ed.), Naturalizing Epistemology, 2d ed., MIT Press, Cambridge, MA, pp. 1-14.

Kornblith H. 1994b, "Naturalism: Both Metaphysical and Epistemological", Midwest Studies in Philosophy 19, pp. 39-52.

Kornblith H. 2002, Knowledge and its Place in Nature, Oxford University Press, Oxford.

Kovac L. 2007, “Information and knowledge in biology", Plant Signaling and Behavior 2, pp. 65-73.

Kroes P., Meijers A. 2006, "The Dual Nature of Technical Artefacts", Studies in History and Philosophy of Science 37, pp. 1-4.

Kuhn T.S. 1996, The Structure of the Scientific Revolutions, University of Chicago Press, Chicago.

Laudan L. 1987, "Progress or rationality? The prospects for normative naturalism", in American Philosophical Quarterly 24, pp. 19-31.

Laudan L. 1990, "Normative naturalism", in Philosophy of Science 57, pp. 44-59.

Loewer B. 1997, "A Guide to Naturalizing Semantics", in B. Hale and C. Wright (eds.), A Companion to the Philosophy of Language, Blackwell, Malden, MA:, pp. 108-126.

MacLaurin J., Dyke H. 2012, "What is analytic metaphysics for?", Australasian Journal of Philosophy 90, pp. 291-306.

Maddy P. 2007, Second Philosophy. A Naturalistic Method, Oxford University Press, Oxford. 
Nagel E. 1956, "Naturalism reconsidered", in Proceedings and Addresses of the American Philosophical Association, 28, pp. 5-17.

Papineau D., 2009: Naturalism, in «The Stanford Encyclopedia of Philosophy», <http://plato.stanford.edu/archives/spr2009/entries/naturalism/>.

Peierls R. 1986, in P.C.W.Davies, J.R. Brown (eds.), The Ghost in the Atom, Cambridge University Press, pp. 70-82.

Quine W.V.O. 1969, Ontological Relativity and Other Essays, Columbia University Press, New York.

Quine W.V.O. 1986, Reply to Morton White, in L. Hahn, P. Schilpp (eds.), The Philosophy of W.V.O. Quine, The Library of Living Philosophers, Open Court, La Salle.

Radder H. 2009, "Why Technologies are Inherently Normative", in D. Gabbay, P. Thagard, J. Woods (eds.), Handbook of the Philosophy of Science, Vol. 9 (ed. by A. Meijers): Philosophy of Technology and Engineering Sciences, Elsevier, Amsterdam, pp. 887-921.

Ramstead M.J.D 2015, "Naturalizing what? Varieties of naturalism and transcendental phenomenology", Phenomenology and the Cognitive Sciences 14, pp. 929-971.

Rysiew, P. 2016, "Naturalism in Epistemology", The Stanford Encyclopedia of Philosophy (Summer 2016 Edition), Edward N. Zalta (ed.), URL $=<$ http://plato.stanford.edu/archives/sum2016/entries/epistemology-naturalized/>.

Sellars W. 1963, Science, Perception and Reality, Routledge \& Kegan Paul Ltd; London, and The Humanities Press: New York, 1963.

Siegel H. 1990, "Laudan's normative naturalism", in Studies in History and Philosophy of Science 21, pp. 295-313.

Sklar L. 2001, "Naturalism and the Interpretation of Theories", Proceedings and Addresses of the American Philosophical Association, 75, pp. 43-58.

Stein H. 1993, "On Philosophy and Natural Philosophy in the Seventeenth Century", Midwest Studies In Philosophy 18, pp. 177-201.

Suppe F., 1989, The Semantic Conception of Theories and Scientific Realism, University of Illinois Press, Chicago. 
Is Science Really What Naturalism Says it is?

Van Fraassen B. 1980, The Scientific Image, Clarendon, Oxford.

Wright W. 2007, "Why Naturalize Consciousness?", Southern Journal of Philosophy 45, pp. 583-607. 